\title{
The outskirts of spiral galaxies: probing stellar migration theory
}

\author{
Judit Bakos and Ignacio Trujillo
}

Instituto de Astrofísica de Canarias Spain, email: bakosjud@gmail.com

\begin{abstract}
One of the most outstanding predictions of the radial migration theory was a progressive increase in the age of the stellar populations toward the galaxy outskirts. This gradual change in the age is caused by a net flux of old stars formed in the inner disk moving toward the outskirts. Thanks to the fact that the age of stellar populations can be interpreted in terms of colors, this prediction was confirmed observationally a few years ago with deep surface brightness profiles of a large sample of nearby galaxies. Our group has now taken a step forward on this as we have explored the properties of the stellar populations beyond the star formation threshold using ultra deep data from SDSS Stripe82. These data allowed us to study the faint outskirts of disks in detail down to a surface brightness level of $30 \mathrm{mag} / \mathrm{arcsec} 2$ in the r'-band. At these surface brightness levels spiral galaxies reveal new and exciting structural components which could not have been seen by regular SDSS imaging: outer disks extend farther out, tidal streams and satellites become visible. We will present deep color profiles of the regions of the galaxies where the disks starts to be confused with the stellar haloes. We confront these colors with model predictions. For some galaxies, the very outer regions are so red that conventional IMFs can not explain their colors. We will discuss whether our new results can be explained within the radial migration scheme.
\end{abstract}

